\title{
Preoperative Care of Laryngoscope Facilitated Vocal Polyp Excision under General Anesthesia
}

\author{
Cuiping Feng*, Lanfang Chen and Jianqiong Wang \\ Yangjiang people's hospital, Yangjiang, Guangdong 529500, China
}

\begin{abstract}
Objective: To study the nature of preoperative nursing care of a Laryngoscope facilitated vocal polyp excision procedure using general anesthesia. Methods: Clinical data from 238 cases of the aforementioned laryngoscope procedure from May 2012 to May 2014 in our hospital's Otolaryngology department was collected. Psychological nursing was administered including preoperative preparation, postoperative monitoring, close observation of vital signs, post-operative nursing, diet, care, and also enhanced nursing skills such as discharge guidance. Results: 238 cases of vocal cord polyp patients were all completely cured and discharged, there was no postoperative infection and other complications. After reviewing the condition of the vocal cords, primarily if the sutures at the incision are fine, there is apparent improvement. Conclusion: The laryngoscope facilitated vocal polyp excision under general anesthesia procedure can deliver satisfying Results when coupled with close patient observation and improved understanding of diseases by the preoperative nurses.
\end{abstract}

\author{
KEYWORDS \\ Laryngoscope \\ Vocal cord polyp \\ Preoperative care
}

\section{Introduction}

Vocal cord polyp is a chronic inflammatory disease of the throat, it refers to the benign laryngeal neoplasm that occurs either unilaterally or bilaterally on the edge of the vocal cord (one-third of it usually occurs on or before the vocal cords). Long-term, excessive strain of the vocal cord are major causes of vocal cord polyps. Smoking and drinking alcohol has often been the main causes of the disease. Main symptoms of aphonia are wheezing and breathing difficulties. This disease is common in otolaryngology and seriously affects pronunciation. The main treatment is the surgical removal of the polyps to improve pronunciation [1]. Vocal polyp excision surgery facilitated by the laryngoscope is a low-risk and high precision procedure with minimum room for trauma. The procedure is systematic and recovery is quick [2]. 238 cases of this procedure from May 2012 to May 2014 in our hospital have achieved satisfactory results.

\footnotetext{
Copyright $(2015$ Cuiping Feng et al.

doi: $10.18686 /$ jn.v4i3.5

Received: July 11, 2015; Accepted: August 5, 2015; Published online: August 21, 2015

This is an open-access article distributed under the terms of the Creative Commons Attribution Unported License (http://creativecommons.org/ licenses/by-nc/4.0/), which permits unrestricted use, distribution, and reproduction in any medium, provided the original work is properly cited. ${ }^{*}$ Corresponding author: Yangjiang people’s hospital, Yangjiang, Guangdong 529500, China. E-mail: fengcuiping123y@sina.com
}

\section{Clinical data}

\subsection{General information}

From May 2012 to May 2014, out of 245 vocal cord polyp cases, 238 patients underwent laryngoscope facilitated downward vocal cord polyp excision. 171 cases were men (accounting for $72 \%$ ), 67 cases were women (accounting for $28 \%$ ), ranging from 28 to 71 years old, the average being 44 years old. The duration of the disease varied from 2 months to 5 years. Hospitalization duration varied from 4 to 12 days. A postoperative pathologist confirmed that they had vocal mucosa polyps.

\subsection{Methods}

All 238 patients underwent the surgery with general anesthesia. Intramuscular luminal injection $(0.1 \mathrm{~g})$ and atropine $(0.5 \mathrm{mg})$, which inhibits respiratory glands, were administered 30 minutes before the surgical procedure. After undergoing a general anesthesia, a parallel vocal cord incision was made at the site of the vocal cord chamber above the throat, and vocal cords mucous membrane was separated from the superficial lamina. If the area of incision showed signs of mucosa edema, the surrounding tissue was removed and sent to the pathology department for further investigation $[3,4]$.

\subsection{Results}

The 238 patients with vocal cord polyp were all cured and 
discharged with no postoperative infection and other complications. After reviewing the effects of the procedure, it is observed that their conditions are improved.

\section{Nursing \\ 3.1. Preoperative nursing \\ 3.1.1. Psychological nursing}

All procedures should be carried out under general anesthesia as patients may feel stressed, anxious and scared. The nurses should adopt a sense of responsibility to explain the procedure to the patients. Explaining and making them aware of the steps involved in the surgical procedure is the only way to ease the stress of the patients. Introducing the relevant preoperative and postoperative surgical considerations encourages the patients to actively cooperate with the surgery. The implementation of psychological nursing guidance in surgery is crucial to the whole process of patient care [5].

\subsubsection{Oral care}

This includes the examination of the oral cavity and throat. The patients who have a history of acute throat inflammation should suspend surgery. Nurses should ensure that the patients gargle saline solution three times a day, the day before the operation. They should also quit smoking and wine.

\subsubsection{Preoperative preparation}

Patients underwent various medical examination prior to the surgery and were told to start fasting at 22:00 on the night before the surgery. On the preoperative night, sedatives should be given, to assure that they get enough sleep. On the morning of the operation, the urethral catheter should be kept in accordance with the doctor's advice, the venous channel should be established and a subcutaneous luminal injection of $0.1 \mathrm{~g}$ and $0.5 \mathrm{mg}$ of atropine should be administered to the patients 30 minutes before the operation. According to the requirements of general anesthesia, emergency equipment should be prepared such as the sputum suction device, sputum suction tube, oxygen pipeline, sputum cup along with a paper and pen to do nursing records [6].

\subsubsection{Preoperative tasks}

The patients should be told to pay attention to oral hygiene. They should use chloride when necessary to keep the oral cavity clean and prevent oral cavity infection. They should also try to avoid talking for 1 week. The patients should explained the purpose and significance of being silent for a week. They can get a pen and paper ready which can be used to express discomfort and demand [7].

\subsection{Postoperative nursing}

\subsubsection{General care}

Patients with general anesthesia should lie recumbent for 6 hours without a pillow, having the head to one side, to allow the mouth secretions and blood flow naturally. The respiratory tract should be unobstructed to prevent blood into the respiratory tract and prevent asphyxia or breathing difficulties. Anti-inflammatory, hemostatic and atomization treatment should be given, ECG monitoring should also be done to monitor normal breathing, pulse, heart rate and the change of blood oxygen saturation, until the patients regain full consciousness and vital signs are normal [8].

\subsubsection{Close observation of vital signs}

According to the nursing care routine, postoperative patients should lie recumbent without a pillow, head to one side, for 6 hours, a low flow oxygen should be administered for 6 hours. ECG monitoring should also be done for 12 hours. Changes or fluctuations of heart rate and blood pressure should be carefully observed. Attention should be paid to any signs of bleeding due to surgical shock. Respiratory changes and breathing difficulties should be closely observed in case of vocal cord edema. The doctor should be informed immediately. An intravenous drip of antibiotics and the right amount of dexamethasone and hemostatic should be administered based on the doctor's prescription [9].

\subsubsection{Post-operative diet}

After 6 hours the patient can have a cold liquid diet. They can eat small portions and slowly swallow the food. They should avoid the liquid from flowing into the throat, this could cause choking. After being in the cold semi-liquid diet for three days, they can switch to a light, digestible diet rich in vitamin A, B and C. The mouth should be kept clean. The mouth should be rinsed with cold water or normal saline after a meal.

\subsubsection{Silence period nursing}

Postoperative patients need to keep silent for 7 days because early postoperative activity can cause discomfort in the unhealed wound and influence the treatment. At the same time, it can cause remission. The nurse should be actively concerned about the patient, teach patients to communicate in sign language or by writing. After one month, they should minimize talking as much as possible, which is conducive to good vocal recovery [10].

\subsubsection{Local nursing}

Observation of bleeding wounds: The patient should be asked to spit out secretions from the mouth gently, they should not cough or spit roughly if there is liquid flowing down the throat. The nature of the mouth secretions should be observed to know whether there is presence of blood or not. This to know if there is an incidence of a bleeding wound. Ultrasonic atomizing inhalation: saline $10 \mathrm{~mL}, 80000 \mathrm{U}$ gentamicin and $5 \mathrm{mg}$ dexamethasone, atomization inhalation should be done twice a day to relieve and prevent laryngeal spasms and edema; Oral care: keep 
the mouth clean, rinse if necessary.

\subsubsection{Observation}

(1) Postoperative bleeding: Because of the rich blood present in pharyngeal tissues, carelessness in the surgical process may easily damage the mucosa, resulting in intraoperative and postoperative wound bleeding. Postoperative patients should be carefully observed for bleeding or blood in the phlegm. If pharyngeal area appears swollen, it could be a sign of the wound bleeding. As such, it should be closely observed and reported to the doctor for appropriate action. (2) The breathing difficulty: because the operation may stimulate the epiglottis, vocal cord and laryngeal mucosa it could cause laryngeal edema, epiglottis edema and laryngospasm. Such patients with sudden breathing difficulties should promptly report to doctors for emergency care.

\subsubsection{Prevention of wound adhesion}

One week post-operation, patients should consciously practice deep breathing for 2-5 times to avoid wound adhesion. This is helped by a routine use of drugs in accordance by the doctor's advice.

\subsection{Discharge guidance}

Patients discharged from the hospital should continue atomization inhalation for 2 times a day, lasting for a period of 1 week. Post-operation, the recovery period takes 2 weeks. Nonetheless, this does not mean that patients should be completely silent but they should just speak minimally. Attention should be given to the pronunciation methods. Excessive talking should be avoided after vocal cord polyp surgery to prevent reoccurrence. Regular reviews should be done. If the voice is hoarse, the doctor should be consulted as soon as possible. Patients should also exercise more to prevent themselves from getting sick.

\section{Experience}

Vocal cord polyp is a common disease. No timely treatment or proper care can lead to physical and psychological effects and also bring unnecessary burden for families. Surgical removal of vocal cord polyp is one of the main and effective treatment methods. The laryngoscope which is essentially, microsurgery has advantages like great vision and high resolution. However, the demand of vocal polyp surgery with administration of general anesthesia under the laryngoscope technique is high. In order to ensure the positive effect of the surgical treatment and improve the success rate of surgery, the nurses should be efficient in preoperative preparation. They should engage themselves in close observation and even complete the necessary disease knowledge education. This is to ensure that the postoperative patients have sufficient knowledge to pay attention to aspects of pronunciation, diet and review, to ensure that the surgery has good effects [11]. In addition, strict silence and careful observation are the keys to the success of vocal cord polyp surgery along with the right daily habits to prevent the reoccurrence of the disease. Due to careful nursing, the clinical treatment of the disease has achieved satisfactory results, greatly improving the elementary course of the disease control rate and cure rate.

This data shows that 238 cases of vocal cord polyp patients were all cured and discharged with no postoperative infection and other complications. The vocal cords are neat and the wound closure is good. This shows that a laryngoscope-assisted procedure can provide vocal polyp patients with a satisfactory curative effect. Nonetheless, it is important to closely observe and enhance preoperative nursing procedures, completed by an in-depth knowledge of the condition.

\section{Conflicts of interest}

These authors have no conflicts of interest to declare.

\section{Authors' contributions}

These authors contributed equally to this work.

\section{Reference}

1. Zhong F, Wang T. Practical otolaryngology. Jinan: Shandong Science and Technology Press. 1997;681.

2. Cao C, Huang S. 320 cases of the laryngoscope under general anesthesia in patients with vocal cord polyps enucleation of preoperative care. Contemp Nurse: Special Edition. 2011;(7):79-80.

3. Ji Dong. Experience of the vocal cords polyp surgery method choice. J Huaihai Med. 2007;25(5):411-412.

4. Ma Q and Huang Y. 625 cases of vocal nodules under electronic laryngoscope enucleation experience. J Pract Med. 2008; (24):81

5. Lou Q. Vocal cord polyp excision of preoperative care. $J$ Natl Med. 2008;20(8):8-13.

6. Wei L. Preoperative care of laryngoscope under general anesthesia vocal cord polyp excision. J Minim Invasive Med. 2012;(3):330-331.

7. Ni T. Laryngoscope under general anesthesia vocal cord polyp excision of 60 cases of preoperative nursing experience. J Med Inf. 2014;(29):211-212.

8. Xu C. The laryngoscope under general anesthesia of vocal cords polyp excision preoperative care. J Mod Med. 2011;(6):127-128.

9. Zhang T. 116 cases of vocal fold polyp laryngoscope under general anesthesia with excision preoperative care. $J$ Inn Mong Tradit Chin Med. 2012;42(41):77-79.

10. Tan L, Wei X, Wei M. The laryngoscope under general anesthesia vocal cord polyp excision of preoperative care. China Med Inno. 2013;14(6):91-93.

11. Xie L. Under the laryngoscope vocal polyp excision of preoperative care. J Inn Mong Tradit Chin Med. 2010;14(32):56-58. 